\title{
Bacteriological Study of Patients with Otitis Media Attending Tertiary Care Hospital
}

\author{
K.N. Ravichitra* and U. Srinivasa Rao \\ Department of Microbiology, ASRAM Medical College, Eluru- 534005, West Godavari dist, \\ Andhra Pradesh, India \\ *Corresponding author
}

\section{A B S T R A C T}

\section{Keywords}

Otitis media, Ear swabs, Antibiogram, Antibiotic resistance

Article Info

Accepted: 06 September 2018 Available Online: 10 October 2018
Otitis media is a major health problem and occurs with high incidence and prevalence in both developed and developing countries. This study was undertaken to determine etiological agents of the otitis media and their antimicrobial susceptibility patterns. Ear swabs collected from clinically diagnosed otitis media were subjected to microbiological investigations. Their antibiogram was determined by Kirby-Bauer disc diffusion method. Out of 248 ear swabs processed $198(81.8 \%)$ samples showed growth of various bacteria. $113(53.3 \%)$ gram positive and $99(46.7 \%)$ gram negative bacteria were isolated. This study shows the rate of isolation of various organisms from the ear swabs and their tendency towards antibiotic resistance.

\section{Introduction}

Otitis media is an inflammatory condition of the middle ear (Richard, 2015). It is the most common disease of childhood after viral upper respiratory tract infections (Paul et al., 2015). These upper respiratory tract infections, often viral, are most commonly responsible for the events that lead to otitis media. Such infections impair the function of the Eustachian tube, creating a negative pressure and transduction into the middle ear (John and Duana, 2005).

Bacterial contamination results by reflux from the oropharynx and this leads to further accumulation of fluid and pus, producing an acute or sometimes chronic illness. Acute infections are caused by respiratory viruses and by certain bacteria Streptococcus pneumoniae, Moraxella catarrhalis, Hemophilus influenzae. When the infection becomes chronic, facultative aerobic gram negative bacilli, anaerobic bacteria and fungi assume a prominent role in a polymicrobial infection (Elmer et al., 2006).

Concern is increasing about community strains of MRSA as an emerging etiological agent (Richard, 2015). The infectious and noninfectious complications of otitis media in childhood can result in serious morbidity. These are the major cause of hearing loss throughout the world. The frequency of 
etiologic agents in ear infection and their resistance pattern varies among populations.

Hence this study was undertaken to know the various etiological agents in ear infection and their antimicrobial susceptibility pattern.

\section{Materials and Methods}

This study was conducted over a period of 12 months from January 2017 to December 2017 at Alluri Sitaramaraju Academy of Medical Sciences, Eluru, on 242 clinically diagnosed otitis media cases to determine bacterial etiologies and their antimicrobial susceptibility patterns. Both inpatients and out patients of all age groups and both sexes were included in the study.

Samples were collected aseptically using sterile swabs and transported to the laboratory without delay.

Processing of ear swabs for culture and sensitivity

The ear discharge samples were cultured on MacConkey agar, blood agar and incubated aerobically at $37{ }^{\circ} \mathrm{C}$ for $24-48 \mathrm{hrs}$. Emergent colonies of bacterial pathogen were preliminarily characterized by colony morphology, gram stain and species identification as per the standard microbiological methods. (Patricia, 2014; Gerald Collee et al., 2012) Antibiotic susceptibility pattern done on MHA by Kirby Bauer disc diffusion method according to CLSI guidelines (Franklin et al., 2012).

\section{Results and Discussion}

A total of 242 ear swabs were received for culture and sensitivity during the period of study, of them 64 were inpatients and 178 were outpatients. Of the total 120 (49.6\%) were males and $122(50.4 \%)$ were females. Of them $44(18.2 \%)$ samples showed no growth of organisms and 198 (81.8\%) samples showed growth of various bacteria. $81.3 \%$ of inpatients and $82 \%$ of outpatients showed culture positivity. No major difference was noted among both groups.

\section{Distribution of samples and their positivity (Table 1)}

A total of 212 organisms were isolated from 198 samples. $14(7.1 \%)$ samples in our study showed polymicrobial etiology and 184 $(92.9 \%)$ samples showed monomicrobial etiology.

Our study showed predominance of monomicrobial etiology. Our study showed predominance of gram positive organisms 113 (53.3\%) in comparison with gram negative organisms $99(46.7 \%)$.

Rate of isolation of various organisms shown in chart -1

Otitis media is a common reason for very young children to seek medical treatment. In spite of the widespread use of antimicrobial therapy for otitis, acute mastoiditis remains the most common supparative complication of acute otitis media.

Table.1 Distribution of samples

\begin{tabular}{|l|l|l|l|l|}
\hline & Total samples & Sterile & Culture positive & \% of culture positives \\
\hline Inpatients & 64 & 12 & 52 & 81.3 \\
\hline Outpatients & 178 & 32 & 146 & 82.1 \\
\hline Total & 242 & 44 & 198 & 81.8 \\
\hline
\end{tabular}




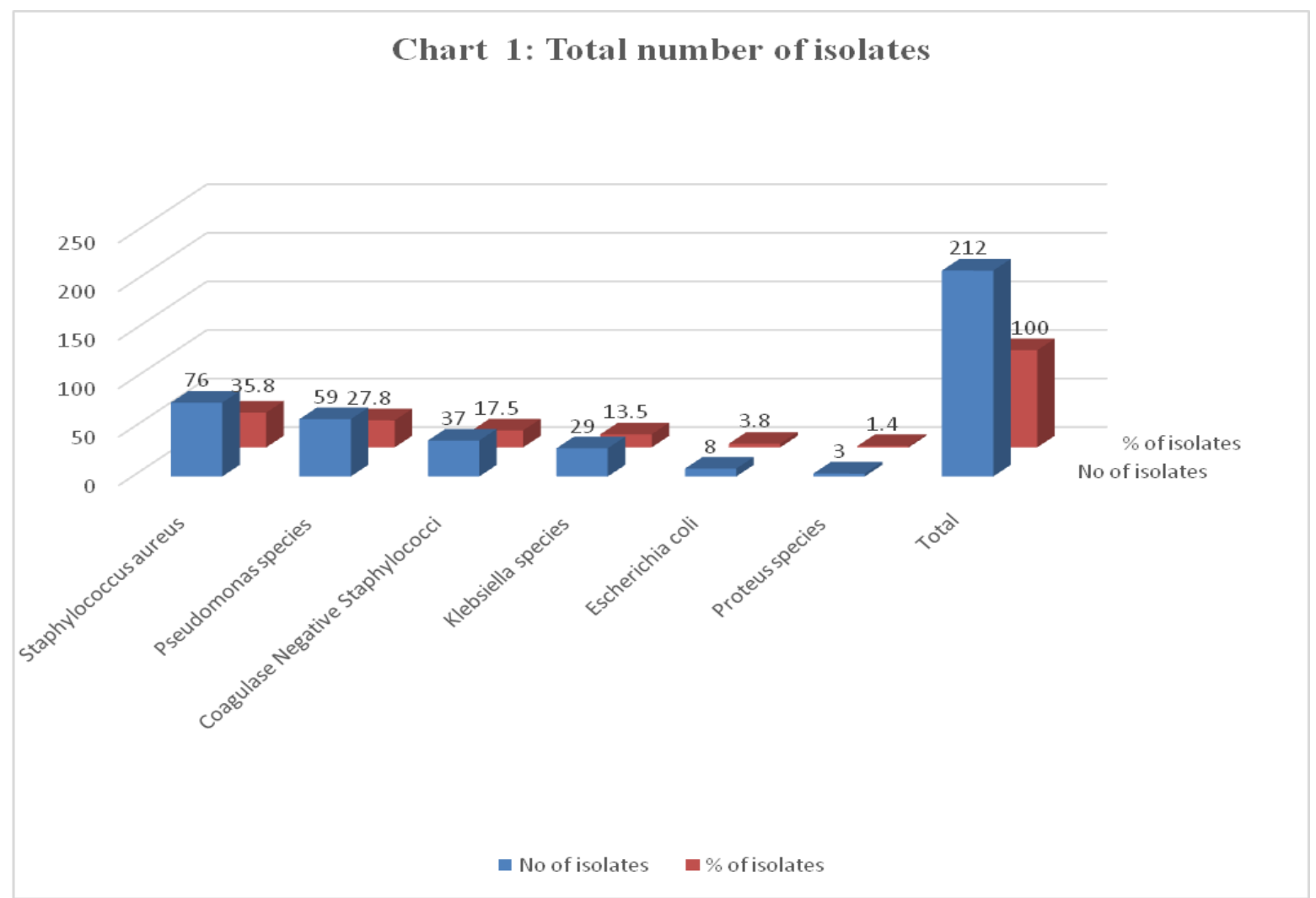

Culture positivity in our study is $81.8 \%$ which is similar to the one reported by Derese Hailu et al., that is $80.4 \%$. (Derese Hailu et al., 2016) Whereas Dagnachew Muluye et al., reported $89.5 \%$ of culture positives, slightly higher than that reported by our study. (Muluye et al., 2013) On the other hand Mohit Agarwal et al., reported only 53.8\% culture positivity. (Agrawal et al., 2017) 7.1\% samples in our study showed polymicrobial etiology and $92.9 \%$ samples showed monmicrobial etiology which is similar to the study conducted by Dagnachew Muluye et al., 9.3\% and 90.7\% (Muluye et al., 2013) and Mesfin Worku et al., 7\% and 94\% (Worku et al., 2014). Our study showed higher isolation of gram positive organisms $53.3 \%$ in comparison with gram negative organisms $46.7 \%$. Staphylococcus aureus is the predominant organism in our study constituting $35.8 \%$ which is similar to the study conducted by Neeti Mishra et al., that is $34.7 \%$ (Neeti Mishra et al., 2014) and Humera Rashid et al., that is $31 \%$ (Humera
Rashid et al., 2014). Pseudomonas was the second common organism isolated in our study constituting $27.8 \%$ which is similar to Neeti Mishra et al., that is $30.4 \%$ whereas Mohit Agarwal et al., reported $65.18 \%$ isolation of Pseudomonas much higher than the one reported by us. Antibiotic sensitivity pattern of gram positive organisms isolated revealed linezolid, amikacin, ciprofloxacin, erythromycin, cefotaxime as the most sensitive antibiotics in that order whereas amoxicillin and clavulinic acid showed high level of resistance to these organisms. Gram negative organisms showed high level of sensitivity to amikacin followed by ciprofloxacin, piperacilin, cefotaxime, whereas amoxicillin clavulinic acid and ampicillin showed higher resistance among organisms.

In conclusion rate of isolation of bacteria was high in patients with otitis media in our study. A predominance of gram positive organisms was noted in comparison to gram negative 
organisms. Isolates showed good sensitivity to amikican and ciprofloxacin. Therefore, culture and susceptibility test is vital for appropriate management of ear infection.

\section{References}

Agrawal M et al., 2017. Bacterial etiology of otitis media and their antibiotic susceptibility pattern among patients coming to a tertiary care hospital, Jaipur, India. J Mahatma Gandhi Univ Med Sci Tec; 2(1):7-9.

Derese Hailu et al., 2016. Pathogenic bacteria profile and antimicrobial susceptibility patterns of ear infection at Bahir Dar Regional Health Research Laboratory Center, Ethiopia. Springer plus journal. 5:466

Elmer W. Koneman et al., 2006. Color Atlas and Textbook of Diagnostic Microbiology. 6th edition. Baltimore: Lippincott Williams Wilkins.

Franklin R. Cockerill et al., 2012. CLSI. Performance Standards for Antimicrobial Disk Susceptibility Tests; Approved Standard: Eleventh Edition. CLSI document M02-A11. Wayne, PA: Clinical and Laboratory Standards Institute.

Humera Rashid et al., 2014. Frequency and Antimicrobial Susceptibility Pattern of Pseudomonas aeruginosa in Ear Swabs. World Applied Sciences Journal, 30 (7): 812-817.

Gerald Collee J., et al., 2012. Mackie \& Mc Cartney: Practical Medical
Microbiology. 14th edition. Churchill Livingstone: Elsevier.

John T. Roehrig, and Duana J. Gubler. 2005. Topley \& Wilson's Microbiology \& Microbial infections | 10th edition: Wilkey. London.

Muluye et al., 2013. Bacterial isolates and drug susceptibility patterns of ear discharge from patients with ear infection at Gondar University Hospital, Northwest Ethiopia. BMC Ear, Nose and Throat Disorders. 13:10.

Neeti Mishra et al., 2014. Prevalence of Micro-Organisms Isolated from Ear Swab. International Journal of Science and Research (IJSR).2118-2120.

Patricia M. Tille. 2014. Bailey \& Scott s Diagnostic Microbiology. 13th edition. St. Louis, Missouri: Mosby Elsevier publications.

Paul W. Flint, Bruce H. Haughey, Valerie Lund, John K Niparko, K. Thomas Robbins, J. Regan Thomas, Marci M. Lesperance. 2015. Cummings Otolaryngology Head \& Neck surgery: $6^{\text {th }}$ edition. Canada. Elsevier Inc.

Richard A. Chole. 2015 Harrison's Principles of Internal Medicine: 19th edition: United States of America. McGraw Hill Medical Companies.

Worku M et al., 2014. Bacterial isolate and antibacterial resistance pattern of ear infection among patients attending at Hawassa university referral Hospital, Hawassa, Ethiopia. Indian $\mathbf{J}$ Otol. 20:155-9.

\section{How to cite this article:}

Ravichitra, K.N. and Srinivasa Rao, U. 2018. Bacteriological Study of Patients with Otitis Media Attending Tertiary Care Hospital. Int.J.Curr.Microbiol.App.Sci. 7(10): 659-662. doi: https://doi.org/10.20546/ijcmas.2018.710.073 